\title{
Symptoms and management of temporohyoid osteoarthropathy and its association with crib-biting behavior in 11 Japanese Thoroughbreds
}

\author{
Yoshihiro SAITO $^{1 *}$ and Tomohiko AMAYA ${ }^{1}$ \\ ${ }^{1}$ Yamatokohgen Animal Medical Clinic, Osaka 583-0867, Japan
}

There have been few reports about temporohyoid osteoarthropathy in Japanese horses. The aim of this study was to describe the symptoms and management of temporohyoid osteoarthropathy and to investigate its association with crib-biting behavior, which is commonly observed in Japanese horses. The data concerning case details, signs, diagnosis procedures, treatment, and outcomes were collected retrospectively from the medical records of 11 Thoroughbreds with temporohyoid osteoarthropathy. The trainers and owners were asked whether the horses had displayed crib-biting behavior. Nine of the horses were diagnosed by guttural pouch endoscopy, and two were diagnosed by skull radiography. Eight horses were treated medically, of which three (37\%) showed improvement; the other three horses underwent ceratohyoidectomy, with two (67\%) showing improvement. Crib-biting behavior was observed in eight (73\%) of the horses. A comparison of data regarding crib-biting behavior among 437 Thoroughbreds from six local riding schools indicated a significant association between temporohyoid osteoarthropathy and crib-biting behavior: the odds ratio for a horse with temporohyoid osteoarthropathy to exhibit cribbiting behavior compared with the general population was 12 (95\% confidence interval, 3-45), and horses exhibiting crib-biting behavior were significantly more likely to have temporohyoid osteoarthropathy ( $P<0.001$, Fisher's exact test). This suggested that cribbiting behavior may be a risk factor for temporohyoid osteoarthropathy.

Key words: crib-biting behavior, guttural pouch endoscopy, temporohyoid osteoarthropathy, Thoroughbred

\author{
J. Equine Sci. \\ Vol. 30, No. 4 \\ pp. 81-85, 2019
}

Temporohyoid osteoarthropathy (THO) affects relatively mature horses and is characterized by various symptoms associated with dysfunction of the facial and vestibulocochlear nerves [11]. It is caused by degenerative changes in the temporohyoid joint (THJ) or by bone proliferation or fracture of the proximal stylohyoid or temporal bone. The symptoms manifest mainly as eyelid paralysis, unilateral ptosis of the muzzle, head tilt and head shaking, or ataxia due to deficits in the facial and/or vestibulocochlear nerves [11]. One study also reported that auditory loss is a common

Received: September 13, 2018

Accepted: September 25, 2019

*Corresponding author. e-mail: yoshihiro00040@hotmail.com (C)2019 Japanese Society of Equine Science

This is an open-access article distributed under the terms of the Creative Commons Attribution Non-Commercial No Derivatives (by-nc-nd) License. (CC-BY-NC-ND 4.0: https://creativecommons.org/licenses/ by-nc-nd/4.0/) neurologic deficit in horses with THO [2]. Diagnosis of THO is based on an examination of skull radiographs, guttural pouch endoscopy, computed tomography (CT), or magnetic resonance imaging (MRI) $[6,11]$. It is commonly treated with intensive medical therapy and ceratohyoidectomy surgery $[3,8,10,11]$. The etiopathology of THO is unknown, although possible causes have been suggested, including infectious otitis media, infectious otitis interna, or a primary degenerative joint disease of the THJ resulting in mechanical stress on the hyoid bone [2]. It has also been suggested that crib-biting behavior may be associated with THO $[5,10]$. Crib biting is a stereotypical behavior in which a horse places its upper incisors on a solid object, arches its neck, and gulps in air [1,5]. This may subject the hyoid apparatus and stylohyoid bone to repeated excessive pressure [5]. Crib-biting behavior is commonly observed in horses in riding clubs in Japan and the U.S.A. [1].

Most of the case studies of THO are from the U.S.A. 
and Europe, and there is little information in the current veterinary literature concerning the background, follow-up, and prognosis for horses with THO in Japan [6]. The aims of this retrospective study were to describe the clinical signs, diagnosis, and prognosis of THO in thoroughbred horses in Japan and to investigate the relationship between crib-biting behavior and THO.

\section{Materials and Methods}

This retrospective study included 11 riding Thoroughbreds that had been diagnosed with THO between 2009 and 2016 at a general equine practice, Yamatokohgen Animal Medical Clinic, in Japan. To obtain diagnostic results of skull radiography and guttural pouch endoscopy, the clinical records of the horses were reviewed. Included in this study were horses with inflammation or osseous proliferation in the region of the THJ or stylohyoid bone.

The radiographic findings were graded from 0 to 3 as follows: grade 0, normal radiographic appearance; grade 1 , mild enlargement of the stylohyoid bone and THJ, with the stylohyoid bone not more than 1.5 times bigger than the normal bone on the other side; grade 2, marked enlargement of the stylohyoid bone and THJ, with the stylohyoid bone being 1.5-2 times bigger than the normal bone on the other side and moderate sclerosis of the temporal bone; and grade 3 , severe enlargement of the stylohyoid bone and THJ, with the stylohyoid bone being 2 times bigger than the normal bone on the other side and severe sclerosis, severe lysis, and/or fractures of the stylohyoid or temporal bone. The endoscopic images were graded similarly: grade 0 , normal endoscopic appearance; grade 1, mild enlargement of the stylohyoid bone and THJ; grade 2, moderate enlargement of the stylohyoid bone and THJ; and grade 3, severe enlargement of the stylohyoid bone and THJ. A published grading system for imaging modalities was used as reference based on the medical records [3].

Information on the clinical progress and outcomes of the horses, and whether they exhibited crib-biting behavior, was obtained from medical records and conversations with their owners and/or trainers. For comparison, data were collecting from six local riding club, including those which kept the horses with THO, regarding the total numbers of horses that exhibited and did not exhibit crib-biting behavior. We obtained data for 437 Thoroughbreds (aged 3-30 years), including geldings, mares, and stallions. These horses did not undergo radiography and endoscopy to detect the presence or absence of THJ abnormalities. We assumed that the horses which did not exhibit any neurological signs of THO did not have any THJ abnormalities. Therefore, they were used as a control group.

The potential association between THO and crib-biting behavior was investigated by calculating the odds ratio of crib-biting behavior among neurological cases of THO (the horses included in the present study) compared with crib-biting behavior in the control group. The proportions of horses with and without crib-biting behavior that had THO were evaluated using Fisher's exact test because the expected number of horses in each cell of the $2 \times 2$ table was $<5 . P<0.05$ was considered to be statistically significant.

\section{Results}

Table 1 summarizes the characteristics, symptoms, examination results, treatments, and outcomes for the 11 Thoroughbred horses. There were two mares, eight geldings, and one stallion, with their median age was 13 years (range, 8-28 years). None of the horses had traveled abroad. The median duration of clinical signs before presentation was 2 days (range, 1-60 days). At presentation, all 11 horses showed facial paralysis; five had corneal ulceration, two had vestibular syndrome, one had torticollis, one had nystagmus, and one had dysphagia. In addition, one horse had a fever of $>39^{\circ} \mathrm{C}$, and another exhibited aspiration pneumonia with dysphagia.

Diagnosis of THO was confirmed by both guttural pouch endoscopy and radiography in seven horses, by guttural pouch endoscopy only in two horses, and by radiography only in two horses. Thickening of the proximal portion of the stylohyoid bone was identified by guttural pouch endoscopy. Bone proliferation of the stylohyoid bone, fusion of the THJ, and sclerosis of the tympanic bulla were noted on skull radiographs. Bone abnormalities on endoscopy and/or radiography were observed on the right side in five horses, the left side in five horses, and bilaterally in one horse.

Treatment was medical $(n=8)$, surgical $(n=1)$, or both medical and surgical $(n=2)$. Medical treatment included antibiotics (penicillin-streptomycin, Mycillin, Meiji, Tokyo, Japan; gentamicin, Gentamax, Ceva Animal Health Pty Ltd., NSW, Australia; ceftiofur, Zoetis Japan, Tokyo, Japan; orbifloxacin, Victas Injection, DS Pharma Animal Health Co., Ltd., Osaka, Japan; enrofloxacin, Baytril, Bayer Yakuhin, Ltd., Tokyo, Japan; sulfamonomethoxine, Daimeton powder, Meiji, Tokyo, Japan) and non-steroidal anti-inflammatory drugs (flunixin meglumine, Banamine Injection 5\%, DS Pharma Animal Health Co., Ltd., or phenylbutazone, Butalone, Apex Laboratories, NSW, Australia), as well as an anti-inflammatory drug (dexamethasone, Dexamethasone Injection, ZENOAQ, Fukushima, Japan), an antioxidant (dimethyl Sulfoxide, Dimethyl Sulfoxide, Kishida Chemical Co., Ltd., Osaka, Japan), and supportive care for keratitis as the primary treatment, based on care previously described in the literature $[10,11]$. Three (37\%) of the eight horses treated medically showed improve- 
Table 1. Summary of the characteristics, signs, treatments, and outcomes for the 11 horses in this study

\begin{tabular}{|c|c|c|c|c|c|c|c|c|c|c|}
\hline Case & Age (y) & Gender & $\begin{array}{l}\text { Acute } \\
\text { onset }\end{array}$ & Cribber & $\begin{array}{l}\text { Affected } \\
\text { side }\end{array}$ & Endoscopic grade & $\begin{array}{l}\text { Radiographic } \\
\text { grade }\end{array}$ & $\begin{array}{l}\text { Clinical and } \\
\text { neurological } \\
\text { signs }\end{array}$ & Treatment & Outcome \\
\hline 1 & 12 & $\mathrm{M}$ & - & - & $\mathrm{R}$ & 1 & Not performed & R CN7 & $\mathrm{AI}, \mathrm{Abx}$ & Euthanized \\
\hline 2 & 17 & M & - & + & $\mathrm{R}$ & 2 & 3 & $\mathrm{R} \mathrm{CN} 7$ & $\mathrm{AI}, \mathrm{Abx}$ & $\begin{array}{l}\text { Return to full } \\
\text { work }\end{array}$ \\
\hline 3 & 18 & G & - & - & $\mathrm{R}$ & 2 & 2 & $\begin{array}{l}\text { R CN7, } \\
\text { dysphagia }\end{array}$ & $\begin{array}{l}\text { AI, NSAID, Abx, } \\
\text { R TT }\end{array}$ & Euthanized \\
\hline 4 & 18 & G & - & + & $\mathrm{R}$ & 2 & 2 & $\mathrm{R} \mathrm{CN} 7$ & $\begin{array}{l}\text { R CHE, AI, NSAID, } \\
\text { Abx, AO }\end{array}$ & $\begin{array}{l}\text { Return to full } \\
\text { work }\end{array}$ \\
\hline 5 & 13 & G & + & + & $\mathrm{L}, \mathrm{R}$ & 3 & Not performed & R CN7, fever & $\mathrm{AI}, \mathrm{Abx}$ & Euthanized \\
\hline 6 & 11 & G & - & + & $\mathrm{L}$ & 1 & 1 & L CN7 & L CHE & $\begin{array}{l}\text { Return to full } \\
\text { work }\end{array}$ \\
\hline 7 & 9 & G & - & + & $\mathrm{R}$ & 3 & 3 & $\mathrm{R} \mathrm{CN} 7, \mathrm{HN}$ & $\mathrm{AI}, \mathrm{Abx}$ & Euthanized \\
\hline 8 & 14 & S & + & + & $\mathrm{L}$ & 1 & 1 & L CN7, 8 & $\begin{array}{l}\text { L CHE, AI, NSAID, } \\
\text { Abx, AO }\end{array}$ & Euthanized \\
\hline 9 & 13 & G & - & + & $\mathrm{L}$ & Not performed & 2 & $\begin{array}{l}\text { L CN7, 8, head } \\
\text { tilt }\end{array}$ & $\mathrm{AI}, \mathrm{Abx}, \mathrm{AO}, \mathrm{TT}$ & Fair \\
\hline 10 & 28 & G & - & - & $\mathrm{L}$ & Not performed & 3 & L CN7, 8 & AI & Good \\
\hline 11 & 8 & G & - & + & $\mathrm{L}$ & 2 & 2 & L CN7, 8 & $\begin{array}{l}\text { AI, NSAID, } \\
\text { Abx, AO }\end{array}$ & $\begin{array}{l}\text { Return to full } \\
\text { work }\end{array}$ \\
\hline Total & $\begin{array}{c}\text { Median } 13 \\
\text { Range 8-28 }\end{array}$ & $\begin{array}{l}\text { G8, M2, } \\
\text { S1 }\end{array}$ & $2 / 11$ & $8 / 11$ & $\begin{array}{l}\text { L5, R5, } \\
\text { LR1 }\end{array}$ & $\begin{array}{c}\text { Median } 2 \\
\text { Range 1-3 }\end{array}$ & $\begin{array}{c}\text { Median } 2 \\
\text { Range 1-3 }\end{array}$ & & & \\
\hline
\end{tabular}

M, mare; G, gelding; S, stallion; R, right; L, left; CN7, facial nerve paralysis; CN8, peripheral vestibular syndrome; HN, horizontal nystagmus; AI, anti-inflammatory drugs (steroids); Abx, antibiotics; NSAID, non-steroidal anti-inflammatory drugs; AO, antioxidants; CHE, ceratohyoidectomy; TT, temporary tarsorrhaphy.

ment: two returned to the same riding use as before after their facial paralysis improved, although one of them was euthanized because of ataxia and astasia approximately 6 years later; the third horse that showed improvement was grazed, and it exhibited slight residual cranial nerve deficits for almost 18 months. The other five horses did not show improvement with medical treatment: three were euthanized because of the persistence or exacerbation of the cranial nerve deficits and corneal ulceration, one was euthanized because of poor prognosis due to aspiration pneumonia and dysphagia, and one could not return to riding because of remaining mild ataxia.

The owners requested three horses to be treated surgically; these horses underwent ceratohyoidectomy on the affected side under general anesthesia. This procedure involves a paramedian submandibular incision to remove the ceratohyoid bone located between the temporohyoid and basihyoid bones [10]. This stops stress from being transmitted from the basihyoid bone to the stylohyoid bone and THJ, thereby preventing aggravation of the symptoms. Two $(67 \%)$ of the three horses treated surgically showed improvement, apart from slight facial paralysis, and returned to competitive dressage and showjumping. Due to hind limb paralysis and astasia during postoperative recovery, the other horse was euthanized. Although this horse was not assessed pathologically, the first examination findings identified moderate enlargement of the left stylohyoid bone and THJ on guttural pouch endoscopy and radiography, as with the other cases. Neurologically, this horse exhibited left facial paralysis, as well as left hind limb paralysis, although this had mostly improved before surgery. The hind limb paralysis during recovery may not have been related to a cranial nerve deficit because slackness or paresis of the penis and anus were observed at the same time.

Of the 11 horses in this study, eight exhibited crib-biting behavior. The data of the control group obtained from six riding clubs showed that 81 out of 463 horses exhibited crib-biting behavior (Table 2). This produced an odds ratio of 12 (95\% confidence interval, $3-45)$ for a horse with THO exhibiting crib-biting behavior, compared with the general population. Horses that displayed crib-biting behavior were significantly more likely to have THO than the other horses (8/89 vs. $3 / 359 ; P=0.0001804$; Fisher's exact test).

\section{Discussion}

The horses with THO in the present study were all Thoroughbreds, as are most horses that are kept in Japan. Their ages, vestibular ataxia, and neurologic symptoms were almost the same as those reported in a previous study $[5,11]$. 
Table 2. Relationship between crib-biting behavior and temporohyoid osteoarthropathy (THO)

\begin{tabular}{lcrrr}
\hline & THO study & Control & Total & \multicolumn{1}{c}{$\begin{array}{l}\text { Odds ratio } \\
(95 \% \mathrm{CI})\end{array}$} \\
\hline Crib-biters & 8 & 81 & 89 & \\
Non-crib-biters & 3 & 356 & 359 & $12(3.0,45)$ \\
\hline Total & 11 & 437 & 448 & \\
Odds & 2.7 & 0.23 & & \\
\hline$P$ - & & & &
\end{tabular}

$P=0.0001804(<0.01)$.

THO can be diagnosed by guttural pouch endoscopy or skull radiography. However, it is thought that guttural pouch endoscopy can detect early changes at a higher rate than skull radiography [11]. It has been suggested that guttural pouch endoscopy should be performed bilaterally because THO may develop on both sides $[2,10]$. The veterinarians who examined each horse decided whether endoscopy, radiography, or both should be conducted to diagnose THO. Therefore, all the horses in the present study did not undergo examination by both procedures. CT and MRI may be able to evaluate osteoarthrosis more accurately; however, we did not use these procedures in this study because there were no facilities in which CT and MRI could be performed to examine the horses near each of the riding clubs $[6,10]$. It is thought that examination using these procedures is necessary for definitive diagnosis.

Ceratohyoidectomy is recommended for the treatment of THO to reduce the physical stress on the stylohyoid bone and THJ [8-10]. When osteoarthrosis develops bilaterally, some researchers have suggested that surgical procedure should be carried out prophylactically for the side that does not present signs [3, 9]. For these horses in the present study, surgical treatment showed better outcomes than medical therapy. This is consistent with previous reports that indicated surgery was a better option than medical therapy alone, which resulted in persistence or deterioration of the paralysis and a significantly lower survival rate [3, $8,10]$. In contrast, one previous study reported generally good outcomes with medical treatment alone [11]; however, the outcomes of the cases treated medically in the present study were not as good as those reported. This difference could be attributed to the fact that the quarter horse was the main breed in that study, and of the 33 affected horses, only 6 were Thoroughbreds [11]. The authors of the abovementioned study may have judged the outcomes of the study more leniently than we did in the present study because not all the horses which resumed their prior use at the time of follow-up returned to athletic use. In addition, most of those horses experienced neurological abnormalities, including residual facial nerve deficit [11]. Although one of the horses that showed improvement after medical treatment returned to work, it developed ataxia after six years and was retired. It was not possible to judge whether aggravation of THO had reoccurred because the horse was not re-examined with radiography and/or guttural pouch endoscopy, and it did not exhibit facial paralysis. It is commonly believed that horses learn crib biting from other affected horses. Epidemiological evidence from previous studies suggested that stereotypy performance in neighboring horses is a contributing factor for development of crib-biting behavior [1]. Further, there are no reported statistically significant differences in the risk of crib biting between various social contact levels [1]. The prevalence of crib-biting behavior in Thoroughbreds is reportedly at least twice that in any other breed [1]. In a population of horses in the U.K. displaying windsucking behavior, the prevalence of colic appeared to be high, and a proportion of these horses reportedly had multiple recurrences of colic [4]. Although some owners and trainers attempt to physically prevent horses from developing crib-biting behavior, their methods (e.g., collars and stable toys) fail to address the underlying cause of these behaviors and may further reduce equine welfare [4]. Crib-biting behavior is very difficult to stop and treat; therefore, owners and trainers should make efforts to prevent the behavior by increasing contact with horses that exhibit crib-biting behavior. The reason why horses exhibit crib-biting behavior should be the focus of future research.

Eight of the 11 cases of THO in this study exhibited cribbiting behavior. It is possible that one of the reasons for the THO may have been stress from the basihyoid bone, which is connected to the root of the tongue, suggesting a causal relationship between crib-biting behavior and THO. A previous study reported that horses with THO were 8 times more likely to show crib-biting behavior than the general population [5]. In the present study, the odds ratio for cribbiting behavior in the horses with THO was 12 compared with the control population, and there was a significant difference in the proportion of horses that had THO between those that exhibited crib-biting behavior and those that did not. Thus, crib-biting behavior may be a risk factor for THO. The control population did not undergo radiography and endoscopy for THO in this study; the control population might have included horses that had any THJ abnormalities and do not exhibit any neurological signs associated with THO. Therefore, our results might be underestimated if there were some horses that had THJ abnormalities in the control group of horses that did not show crib-biting behavior, and they might have been overestimated if there were some horses that had THJ abnormalities in the control group of horses that did show crib-biting behavior.

The lack of association between radiographic and endoscopic grades and outcome for THO has recently been reported [3], and this study further shows that severity of 
radiography and/or endoscopy findings and outcome are not significantly associated. Though we judge that age and presence or absence of crib-biting behavior are not significantly associated, other authors found that there are no significant correlations in the time to develop neurological signs associated with THO between horses that exhibit crib-biting and those that do not [5]. Meanwhile, horses that do not show clinical signs such as facial paralysis have been shown by $\mathrm{CT}$ and histopathology to have undergone age-associated degenerative changes to both the left and right THJ [7]. On the other hand, no studies have investigated horses that do not exhibit crib-biting behavior, and those that exhibit crib-biting behavior have not yet been screened for degenerative changes in subclinical THO via either guttural pouch endoscopy or radiography.

Further studies are needed to determine the relationships between THO, crib-biting behavior, and the hyoid apparatus. The results of these studies may lead us to investigate the causes of THO and prevent its development. In addition, the next step would be studies in which relative evaluations of screening examinations via skull radiography, guttural pouch endoscopy, CT, and MRI are performed in healthy horses that display crib-biting behavior and those that do not.

\section{Acknowledgments}

We would like to thank Drs. M. Furukawa, S. Saito, T. Mizukami, A. Hayami, K. Maeda, and Y. Imamura from the Yamatokohgen Animal Medical Clinic for contributing cases to the retrospective study and supporting the study.

\section{References}

1. Albright, J.D., Mohammed, H.O., Heleski, C.R., Wickens, C.L., and Houpt, K.A. 2009. Crib-biting in US horses: breed predispositions and owner perceptions of aetiology. Equine Vet. J. 41: 455-458. [Medline] [CrossRef]

2. Aleman, M., Spriet, M., Williams, D.C., and Nieto, J.E. 2016. Neurologic deficits including auditory loss and recovery of function in horses with temporohyoid osteoarthropathy. J. Vet. Intern. Med. 30: 282-288. [Medline]
[CrossRef]

3. Espinosa, P., Nieto, J.E., Estell, K.E., Kass, P.H., and Aleman, M. 2017. Outcomes after medical and surgical interventions in horses with temporohyoid osteoarthropathy. Equine Vet. J. 49: 770-775. [Medline] [CrossRef]

4. Escalona, E.E., Okell, C.N., and Archer, D.C. 2014. Prevalence of and risk factors for colic in horses that display crib-biting behaviour. BMC Vet. Res. 10 (Suppl 1): S3. [Medline] [CrossRef]

5. Grenager, N.S., Divers, T.J., Mohammed, H.O., Johnson, A.L., Albright, J., and Reuss, S.M. 2010. Epidemiological features and association with crib-biting in horses with neurological disease associated with temporohyoid osteoarthropathy (1991-2008). Equine Vet. Educ. 22: 467-472. [CrossRef]

6. Inui, T., Yamada, K., Itoh, M., Yanagawa, M., Higuchi, T., Watanabe, A., Imamura, Y., Urabe, M., and Sasaki, N. 2017. Computed tomography and magnetic resonance imaging findings for the initial stage of equine temporohyoid osteoarthropathy in a Thoroughbred foal. J. Equine Sci. 28: 117-121. [Medline] [CrossRef]

7. Naylor, R.J., Perkins, J.D., Allen, S., Aldred, J., Draper, E., Patterson-Kane, J., and Piercy, R.J. 2010. Histopathology and computed tomography of age-associated degeneration of the equine temporohyoid joint. Equine Vet. J. 42: 425-430. [Medline] [CrossRef]

8. Oliver, S.T., and Hardy, J. 2015. Ceratohyoidectomy for treatment of equine temporohyoid osteoarthopathy (15 cases). Can. Vet. J. 56: 382-386. [Medline]

9. Pease, A.P., van Biervliet, J., Dykes, N.L., Divers, T.J., and Ducharme, N.G. 2004. Complication of partial stylohyoidectomy for treatment of temporohyoid osteoarthropathy and an alternative surgical technique in three cases. Equine Vet. J. 36: 546-550. [Medline] [CrossRef]

10. Palus, V., Bladon, B., Brazil, T., Cherubini, G.B., Powell, S.E., Greet, T.R.C., and Marr, C.M. 2012. Retrospective study of neurological signs and management of seven English horses with temporohyoid osteoarthropathy. Equine Vet. Educ. 24: 415-422. [CrossRef]

11. Walker, A.M., Sellon, D.C., Cornelisse, C.J., Hines, M.T., Ragle, C.A., Cohen, N., and Schott, H.C. 2nd. 2002. Temporohyoid osteoarthropathy in 33 horses (1993-2000). $J$. Vet. Intern. Med. 16: 697-703. [Medline] 